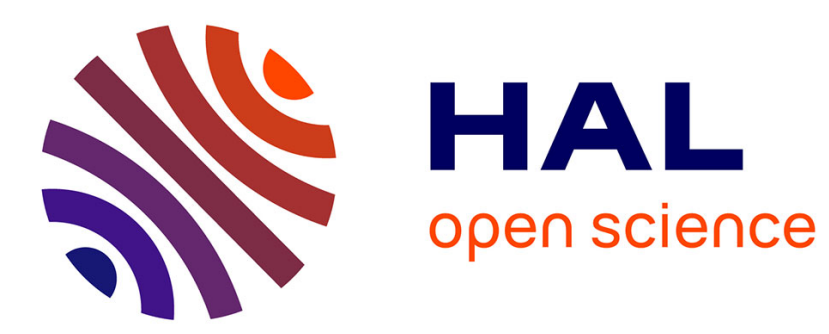

\title{
Kinetics of growth of non-equilibrium fluctuations during thermodiffusion in a polymer solution
}

Marina Carpineti, Matteo Sabato, Fabrizio Croccolo, Alberto Vailati

\section{To cite this version:}

Marina Carpineti, Matteo Sabato, Fabrizio Croccolo, Alberto Vailati. Kinetics of growth of nonequilibrium fluctuations during thermodiffusion in a polymer solution. European Physical Journal E: Soft matter and biological physics, 2019, 42 (2), 10.1140/epje/i2019-11786-x . hal-02473074

\section{HAL Id: hal-02473074 https://hal.science/hal-02473074}

Submitted on 12 Feb 2020

HAL is a multi-disciplinary open access archive for the deposit and dissemination of scientific research documents, whether they are published or not. The documents may come from teaching and research institutions in France or abroad, or from public or private research centers.
L'archive ouverte pluridisciplinaire $\mathbf{H A L}$, est destinée au dépôt et à la diffusion de documents scientifiques de niveau recherche, publiés ou non, émanant des établissements d'enseignement et de recherche français ou étrangers, des laboratoires publics ou privés. 


\title{
Kinetics of growth of non-equilibrium fluctuations during thermodiffusion in a polymer solution
}

\author{
Marina Carpineti ${ }^{1}$, Matteo Sabato ${ }^{1}$, Fabrizio Croccolo ${ }^{2}$, and Alberto Vailati ${ }^{1}$ \\ 1 Dipartimento di Fisica, Università degli Studi di Milano, I-20133 Milano, Italy \\ 2 Laboratoire des Fluides Complexes et leurs Réservoirs, IPRA, UMR5150 E2S-Université de Pau et des Pays de l'Adour, \\ CNRS, TOTAL, F-64600 Anglet, France
}

Received: date / Revised version: date

\begin{abstract}
A thermal diffusion process occurring in a binary liquid mixture is accompanied by long-ranged non-equilibrium concentration fluctuations. The amplitude of these fluctuations at large length scales can be orders of magnitude larger than that of equilibrium ones. So far non-equilibrium fluctuations have been mainly investigated under stationary or quasi-stationary conditions, a situation that allows to achieve a detailed statistical characterization of their static and dynamic properties. In this work we investigate the kinetics of growth of non-equilibrium concentration fluctuations during a transient thermodiffusion process, starting from a configuration where the concentration of the sample is uniform. The use of a large molecular weight polymer solution allows to attain a slow dynamics of growth of the macroscopic concentration profile. We focus on the development of fluctuations at small wave vectors, where their amplitude is strongly limited by the presence of gravity. We show that the growth rate of non-equilibrium fluctuations follows a power law $R_{f}(q, t) \propto \frac{1}{t}$ as a function of time, without any typical time scale and independently from the wave vector. We formulate a phenomenological model that allows to relate the rate of growth of non-equilibrium fluctuations to the growth of the macroscopic concentration profile in the absence of arbitrary parameters.
\end{abstract}

PACS. XX.XX.XX No PACS code given

\section{Introduction}

Diffusion processes in liquid mixtures under the action of a temperature or concentration gradient are accompanied by giant non-equilibrium concentration fluctuations $[1,2]$. These fluctuations have been extensively described and investigated in theoretical and experimental studies performed under ideal conditions, typically during a thermal diffusion process at steady-state $[1,3,4]$ or during quasistationary processes, such as free diffusion [5-8]. A key open question is how do the fluctuations develop in a binary liquid mixture starting from a condition of uniform concentration and leading to a steady macroscopic concentration profile. The kinetics of growth of the concentration gradient under these conditions is of great relevance for the determination of the transport coefficients of mixtures. A huge effort has been devoted to the development of methods suitable to determine the time evolution of the macroscopic concentration gradient in multi-component mixtures. This includes the recent development of molecular dynamics simulations, which have been used to determine the transport coefficients during a thermodiffusion process in a binary mixture [9]. Of particular rele-

Send offprint requests to: marina.carpineti@unimi.it vance is the investigation of the transport coefficients of ternary mixtures in the absence of gravity on the International Space Station (ISS), performed in the framework of the SODI-DCMIX project of the European Space Agency [10-13].

Conversely, so far the investigation of the growth of fluctuations during the development of the macroscopic state has been very limited. A theoretical model based on Fluctuating Hydrodynamics provides an effective description of the statistical properties of the non-equilibrium fluctuations (NEFs) for systems where the macroscopic state changes much slower than the fluctuations [14]. Moreover, recent simulations [15] describe the onset of nonequilibrium fluctuations induced by thermophoretic diffusion in microgravity, predicting a spinodal-like growth [1619] of the fluctuations, leading to a peaked structure factor with scale invariance at large wave vectors. A difficulty in investigating transient diffusion processes on Earth is that gravity strongly affects the behaviour of long wavelength fluctuations, limiting their amplitude below a typical cut-off size, and making it difficult to observe a peaked structure factor. In principle the effect of gravity can be reduced by using a thin sample [20], but this choice limits the amplitude of the light scattered by non-equilibrium fluctuations, thus making difficult to characterize them 
during a transient diffusion process. Of course another option is to perform experiments in microgravity conditions and this is one of the purposes of the Giant Fluctuations (formerly NEUF-DIX) project of ESA [21]. The present work performed on earth is intended as preliminary to the future tests in microgravity conditions. We present the results of a study performed on Earth at the onset of non-equilibrium concentration fluctuations induced by the Soret effect in a solution of a polystyrene polymer in toluene. Transient processes are usually very fast and it is difficult to collect a sufficient amount of data to obtain a good statistical characterization of them. As suggested in [15], a more accurate statistics can be achieved by increasing the time necessary for the development of a concentration gradient induced by thermophoresis. To slow down the kinetics we chose to use a solution of a high molecular weight polymer. The concentration of polymer is chosen above the overlap concentration to guarantee that the amplitude of the scattered light is large enough to be detected.

We use a quantitative shadowgraph technique $[22,6$, 7 , particularly effective for studying this kind of phenomena due to its extremely high sensitivity at small wave vectors. Shadowgraph relies on the detection in the near field of the interference between the transmitted beam and the scattered light. Depending on their wave vector, concentration fluctuations produce changes of the index of refraction, which appear as modulations of light intensity on the images. With respect to light scattering, Shadowgraph makes it possible to directly visualize the sample, but also to recover the structure factor from the images, collecting light at small wave vectors $q$ without problems of stray light rejection and alignment that affect small angle light scattering. The only disadvantage of the technique is the presence of a strongly $q$-dependent transfer function, which usually requires a precise calibration to determine the static structure factor of the fluctuations (See for example ref [23]).

In this work we have focused our attention on the study of the kinetics of growth of non-equilibrium concentration fluctuations. As we will show, the method that we have used allows to get rid of the shadowgraph transfer function without performing calibration measurements.

We will show that the growth rate of concentration fluctuations exhibits a nearly $q$-independent power law behaviour $R_{f}(t) \propto t^{-\alpha}$, at variance with the diffusive growth of the amplitude of the concentration modes $c(q, t) \propto$ $1-\exp \left(-D q^{2} t\right)$, whose characteristic time strongly depends on the wave vector.

\section{Non-equilibrium fluctuations in diffusion processes}

NEFs play a fundamental role in all diffusive processes [1] due to the fact that they perturb significantly the macroscopic state. Indeed, it can be shown that the microscopic mass flows generated by non-equilibrium fluctuations are responsible for the Fickean diffusive mass transfer [24]. Moreover, it has been predicted recently that non-equilibrium fluctuations can significantly modify the interactions between macroscopic bodies embedded in a fluid [25-29], a result that has not yet been confirmed experimentally. Linearized fluctuating hydrodynamics gives a quite complete theoretical description of NEFs in binary liquid mixtures when ideal conditions are realized, as for example stationary states, small gradients, and diluted systems $[1,30-33]$. Theoretical work provided evidence that the non-equilibrium fluctuations are long range correlated, their static structure factor diverging as $q^{-4}$ as the wave vector $q$ goes to zero. At large wave vectors the fluctuations relax diffusively and they are not affected by gravity. However, at small wave vectors gravity frustrates fluctuations, because at these length scales buoyancy determines a faster relaxation than diffusion [34]. The separation between these two regimes of relaxation is reflected by the presence of a roll-off in the static structure factor of the non-equilibrium fluctuations at the roll-off wave vector [14]:

$$
q_{r o}=\left(\frac{\beta g \nabla c}{\nu D}\right)^{\frac{1}{4}}
$$

where $g$ is the acceleration of gravity, $c$ is the weight fraction of the denser component $\nu$ the kinematic viscosity, $D$ the diffusion coefficient and $\beta$ the solutal expansion coefficient. For $q<q_{R O}$ the structure factor is almost constant. These predictions found confirmation in many experiments performed on Earth [3,4,6,7,35-39]

Further experiments allowed to establish that under microgravity conditions the only limit to the growth of non-equilibrium fluctuations is determined by the size of the cell containing the sample, which imposes a finite size limit $[23,15,40]$. Notwithstanding the good understanding of non-equilibrium fluctuations at steady state in the linear regime, recent experiments have shown that a deeper understanding of non-equilibrium fluctuations under more general conditions would be highly desirable $[8,15,41,42]$.

Our attention is focused on the determination of the kinetics of growth of the concentration fluctuations' modes during the transient leading to a stationary state and on the comparison of the results with theoretical predictions and preliminary experimental data $[14,15]$. Starting from a condition where the concentration is uniform, non-equilibrium fluctuations can be induced by taking advantage of a thermodiffusion process. When a temperature gradient $\nabla T$ is suddenly applied to a liquid mixture, previously kept at fixed temperature, a mass flow $j=-\rho D\left[\nabla c+S_{T} c(1-c) \nabla T\right]$ is induced due to Soret effect, until a steady state is reached when a macroscopic constant concentration gradient $\nabla c_{s}=-S_{T} c(1-c) \nabla T$ is formed inside the sample. Assuming that the system is in a gravitationally stable configuration, it is possible to describe how the concentration profile evolves in time, writing it as a function of the dimensionless height $\zeta=z / a$ and time $t[14,43]$ (Fig.1) : 


$$
\begin{aligned}
c(\zeta, t)=c_{0} & +a \nabla c_{s}\left\{\frac{1}{2}-\zeta-\frac{2}{\pi^{2}} \sum_{j=1}^{\infty} \frac{1}{j^{2}}\left[1-(-1)^{j}\right]\right. \\
& \left.\times \cos (j \pi \zeta) \exp \left(-\frac{j^{2} \pi^{2} D}{a^{2}} t\right)\right\}
\end{aligned}
$$

where $z$ is the vertical coordinate, $a$ is the vertical thickness of the sample and $c_{0}$ is the initial uniform concentration. Theory and preliminary experiments performed on Earth show that during a transient thermal diffusion process the static structure factor of concentration fluctuations has features similar to those observed at steady state, namely a $q^{-4}$ decay at large $q$ vectors and a rolloff at smaller wave vectors. It can be shown [14] that the structure factor can be written as

$$
S(q, t)=S_{e q}\left[1+\left(\frac{\nabla c(t)}{\nabla c_{g}}-1\right) \frac{1}{1+\left(\frac{q}{q_{r o}(t)}\right)^{4}}\right]
$$

where $S_{e q}$ is the equilibrium static structure factor, $\nabla c_{g}$ is the equilibrium concentration gradient induced by sedimentation, and $q_{\text {ro }}(t)$ is the roll-off wave vector defined in Eqn. 1, where now the concentration gradient $\nabla c$ is time dependent due to the transient condition.

Notice that at the impermeable horizontal boundaries the mass flow must be zero. Therefore, following Archibald $[44,14]$, the concentration gradient at the boundaries must reach instantaneously its steady state value $\nabla c_{s}$. As a consequence, the concentration profile shown in Fig. 1 can be roughly divided into three regions: two boundary layers where the gradient has achieved approximately the steady state value $\nabla c_{s}$ and one central portion where the gradient is zero. Since the structure factor is proportional to $\nabla c$ below $q_{\text {ro }}$ and to $\nabla c^{2}$ at larger wave vectors (see Eqn. 1) the only regions where its amplitude is significantly large are the boundary layers.

Under typical experimental conditions $S(q) \gg S_{e q}$ and $\nabla c \gg \nabla c_{g}$ and the structure factor of fluctuations occurring in the boundary layers can be approximated as

$$
S(q)=S_{e q}\left(\frac{\nabla c_{s}}{\nabla c_{g}}\right) \frac{1}{1+\left(\frac{q}{q_{r o}}\right)^{4}}
$$

Equation 4 does not depend on time. However, in any real light scattering and shadowgraph experiment the structure factor is integrated across the sample thickness. As outlined above, in a transient thermal diffusion experiment only the boundary layer regions contribute significantly to it, and their growth gives rise to a time dependence of the integrated structure factor $S_{c}(q, t)$ :

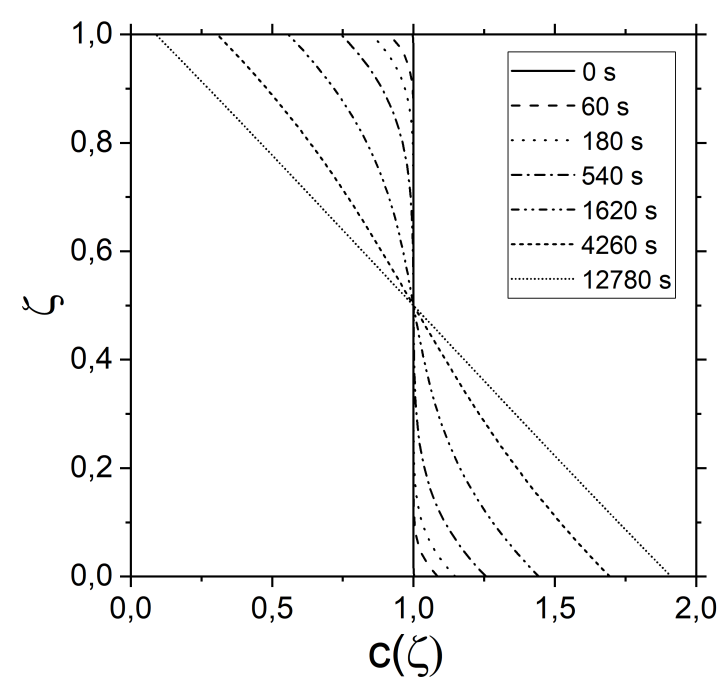

Fig. 1. Time evolution of the concentration profile plotted vs the normalized height $\zeta$ in a thermal diffusion process. Concentration profiles have been obtained numerically from Eqn. 2

$$
\begin{aligned}
& S_{c}(q, t)= \\
& =S_{e q}\left(\frac{\nabla c_{s}}{\nabla c_{g}}\right) \frac{1}{1+\left(\frac{q}{q_{r o}}\right)^{4}}\left(\int_{0}^{\delta(t)} d z+\int_{a-\delta(t)}^{a} d z\right)= \\
& =S_{e q}\left(\frac{\Delta c(t)}{\nabla c_{g}}\right) \frac{1}{1+\left(\frac{q}{q_{r o}}\right)^{4}}
\end{aligned}
$$

Here $\delta(t)$ is the boundary layer thickness and $\Delta c(t)=$ $2 \nabla c_{s} \delta(t)$

From Eqn. 5 it can be appreciated that the structure factor is proportional to the difference between the concentrations at the two cell boundaries $\Delta c$ (see Fig. 1) increases in time. On the contrary, the roll-off position is almost stationary, because it depends on the magnitude of the concentration gradient near the boundaries, which reaches the steady-state almost immediately after the beginning of the process [14].

A different behavior is predicted for measurements performed under microgravity conditions starting from a configuration where the concentration is uniform [15]. In the absence of buoyancy driven effects, the structure factor is expected to exhibit a peak at finite wave vector that moves in time to smaller $q$ vectors, while its amplitude increases. This behaviour is strongly reminiscent of that observed during spinodal decomposition processes, where a dominant mode in the concentration flutuations depends on the fact that the process is driven by a negative diffusion coefficient $[16-18,15]$. For the growth of NEFs, however, the presence of a dominant mode in the fluctuations is originated by the competition between the diffusive and Soret fluxes [15]. During the transient, the mass flow is 
dominated by the Soret flux, which gives rise to a partial demixing of the sample, a situation that mirrors, at least qualitatively, the demixing process occurring during a spinodal decomposition. Some preliminary results collected in microgravity show fair agreement with the theoretical predictions [15]. However, in order to confirm the presence of a peak at small wave vectors, further efforts are required.

\section{Material and methods}

The cell is formed by two sapphire windows pressed against an o-ring gasket, $26 \mathrm{~mm}$ inner diameter, delimiting the sample volume, while two spacers of thickness $1.3 \mathrm{~mm}$ placed between the windows guarantee a well defined sample thickness. The sample is thermalized using two ring shaped Peltier elements thermally coupled to the windows, and controlled through two Proportional-IntegralDerivative servo-controls with an accuracy of $0.01^{\circ} \mathrm{C}$.

Two needles inserted in the o-ring allow to fill the cell. In filling the cell it is important to carefully remove any residual air, as the presence of bubbles strongly affects the thermal transport through the sample. The filled cell is carefully leveled horizontally, after checking that all the optical components are well aligned along the vertical optical axis of the setup.

The onset of the fluctuations is a quite elusive process and in order to study it, it is very important to slow down the kinetics of the process. For this purpose we chose to use a polymer with large molecular weight. $M_{w}=7.06 \times 10^{5} \mathrm{~g} \mathrm{~mol}^{-1}$. The polymer is diluted in toluene with a mass fraction $w=0.02$, corresponding to a concentration $c_{0}=0.0173 \mathrm{~g} \mathrm{~cm}^{-3}$ (approximately three times the overlap concentration $\left.c_{o}=0.0057 \mathrm{~g} \mathrm{~cm}^{-3}\right)$ and to a collective diffusion coefficient $D=4.3 \times 10^{-7} \mathrm{~cm}^{2} \mathrm{~s}^{-1}$ $[45,46]$. The choice to work above the overlap concentration is dictated by the need of having a trade-off between a slow dynamics and a significant amount of light scattered by the sample. We stress that at this concentration the collective diffusion coefficient, describing the rate at which concentration fluctuations decay, is independent of the molecular mass of the polymer, but is still extremely low. Before starting a measurement cycle, the sample is initially kept at a uniform temperature of $25^{\circ} \mathrm{C}$ for some hours to reach an equilibrium state. To avoid convective motions caused by small residual gradients, we impose a small temperature difference of $0.1^{\circ} \mathrm{C}$ between the two Peltier elements, with the above temperature higher than the below one. We collect images of the sample in the equilibrium state (200 frames at a rate of $1 s^{-1}$ ) to characterize the camera background.

The measurement cycle is started by applying a temperature difference $\Delta T=20^{\circ} \mathrm{C}$, heating the upper face at $35^{\circ} \mathrm{C}$, and cooling the lower one at $15^{\circ} \mathrm{C}$. After the imposition of a temperature difference between the sapphire windows, a constant temperature gradient develops inside the cell with a characteristic time much faster than the one needed to attain a steady concentration profile. This is due to the fact that the thermal diffusivity of the sample is two orders of magnitude larger than its diffusion coefficient. We can estimate a time $\tau \approx 100 s$ for the development of the thermal gradient. The sample has a positive Soret coefficient $S_{T}=0.238 K^{-1}$ [45], and therefore the mass flux is in the direction of the colder temperature, with stabilizing effect with respect to convective motions. When the steady state is approached, the mass flux vanishes leading to a constant concentration gradient $\nabla c_{s}$ across the whole cell. We can estimate the time necessary for the system to reach the stationary state to be about $\tau=a^{2} / \pi^{2} D \approx 11.5$ hours.

We study the fluctuations with a highly sensitive shadowgraph technique $[7,23]$ whose setup is very simple, easy to align and extremely versatile. The light source is a super- luminescent diode (SLD) Superlum SLD 261-MP with wavelength $\lambda=670 \mu \mathrm{m}$ and bandwidth (FWHM) of $7.5 \mathrm{~nm}$. This source is definitely more suitable for a shadowgraph experiment than a laser, as the larger bandwidth prevents fringe formation coming from the interference of beams reflected by optical surfaces. The SLD is coupled to a monomode fiber. An achromatic doublet placed before the sample cell collimates the diverging beam coming out of the fiber.

A scientific CMOS detector (IDT NX4-S1) $13.9 \mathrm{~mm} \times$ $13.9 \mathrm{~mm}$ with a resolution of $1024 \times 1024$ pixels is placed at a distance of $41 \mathrm{~cm}$ from the cell. The collimation of the beam is carefully calibrated to give the exact correspondence between pixels and lengths in real space 1 pixel $=13.68 \mu \mathrm{m}$. One advantage of the shadowgraph diagnostics is that the scattered light is superimposed with the transmitted beam onto the sensor, which receives all the light coming from the cell falling inside the solid angle subtended by the sensing area. The transmitted beam acts as a local oscillator and interferes both with the scattered and the stray light coming from spurious reflections, imperfections in the optics, or dust particles along the optical path. As a results, the intensity of the light collected by the detector is linearly related to the amplitude of the scattered field $I(\mathbf{x}, t) \propto\left|E_{0}\right|^{2}+2 \operatorname{Re}\left\{E_{0} E_{s}^{*}(\mathbf{x}, t)\right\}$, where $E_{0}$ is the amplitude of the transmitted field and $E_{s}(\mathbf{x}, t)$ the scattered field. After the imposition of the temperature gradient to the sample, we start acquiring a series of 900 shadowgraph images with a sampling period of $4 \mathrm{~s}$. The number of images was dictated by the storage capacity of the CMOS camera, while the sampling time was chosen to guarantee a good statistical accuracy in the initial stages of the process. It must be pointed out that the data are intrinsically noisy during the transient and can be reduced only by performing time averages across a time much larger than that needed for the relaxation of the fluctuations. For non-equilibrium fluctuations the slowest mode corresponds to the roll-off wave vector, and for our experimental sample the slowest fluctuations relax with a time constant $\tau_{r o}=\left(D q_{r o}\right)^{-1} \approx 20 \mathrm{~s}$. The reproducibility of the process was tested by performing three independent runs under the same experimental conditions, which yielded comparable results. Each shadowgraph image contains several contributions to the scattered field $E_{s}(\mathbf{x}, t)$ : a static background $E_{B}(\mathbf{x})$, equilib- 


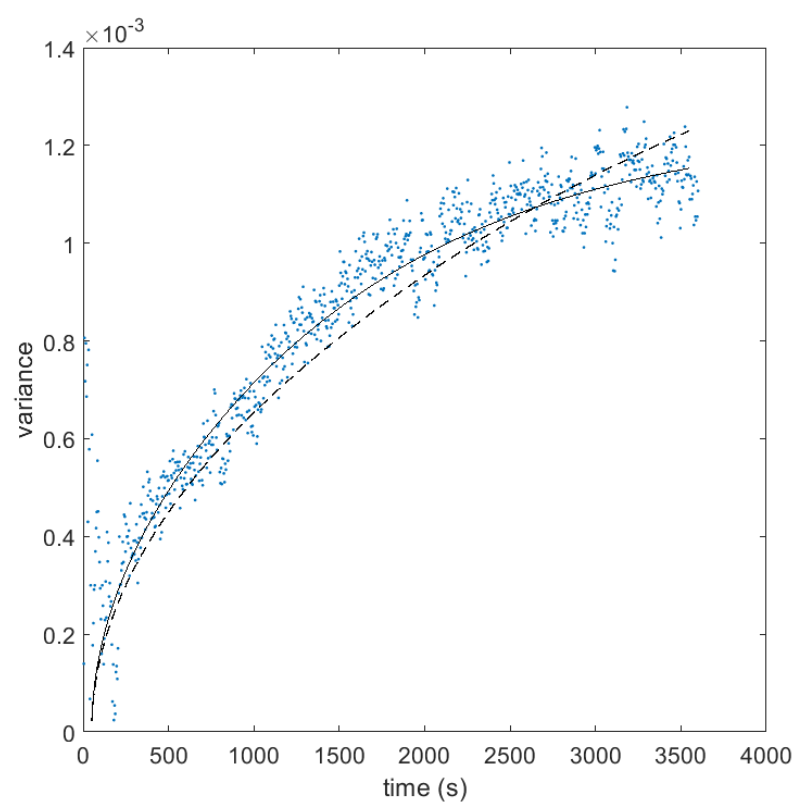

Fig. 2. Time evolution of the variance of shadowgraph images on non-equilibrium concentration fluctuations. The dashed line represents the theoretical time evolution of the concentration difference across the sample obtained from Eqn. 8 using the reference value of the diffusion coefficient $D=4.3 \times 10^{-7} \mathrm{~cm}^{2} \mathrm{~s}^{-1}$. The solid line is the best fit of the experimental data with Eqn. 8 , yielding a value of the diffusion coefficient 2.5 times larger than the reference one.

rium temperature fluctuations $E_{T e}(\mathbf{x}, t)$, equilibrium concentration fluctuations $E_{c e}(\mathbf{x}, t)$, non-equilibrium temperature fluctuations $E_{\text {Tne }}(\mathbf{x}, t)$, non-equilibrium concentration fluctuations $E_{c n e}(\mathbf{x}, t)$ and a dynamic background due to the camera noise $E_{D}(\mathbf{x}, t)$. All the dynamic terms have zero mean.

The average of a set of images $I_{0}(\mathbf{x})=\langle\mathbf{I}(\mathbf{x}, \mathbf{t})\rangle_{\mathbf{t}}$ allows to recover the background term $E_{B}(\mathbf{x})$, which can be eliminated from each image by subtraction so to obtain the normalized intensity distribution [15]:

$$
i(\mathbf{x}, t)=\frac{I(\mathbf{x}, t)-I_{0}(\mathbf{x})}{I_{0}(\mathbf{x})}
$$

\section{Results and discussion}

A first information on the growth of non-equilibrium fluctuations can be obtained from the time evolution of the variance of the shadowgraph images of the fluctuations (Fig. 2) $\operatorname{var}[i(\mathbf{x}, t)]$, which is proportional to the integral of the scattered light over the solid angle subtended by the detector

$$
\begin{aligned}
& \operatorname{var}[i(\mathbf{x}, \mathbf{t})]=\sum_{\mathbf{x}} \frac{i(\mathbf{x}, \mathbf{t})^{2}}{N} \propto \\
& \operatorname{var}\left[E_{\text {Tne }}(\mathbf{x}, t)\right]+\operatorname{var}\left[E_{\text {cne }}(\mathbf{x}, t)\right]+\operatorname{var}\left[E_{D}(\mathbf{x}, t)\right] .
\end{aligned}
$$

Here we have omitted the contributions due to equilibrium fluctuations because under the conditions of interest for our experiment their amplitude is much smaller than that of non-equilibrium fluctuations and can thus be neglected. Following the initial phase where the temperature profile reaches a steady state, the signal starts growing until it finally reaches a plateau. The growth of the variance of the scattered light provides a first evidence of the emergence of the non-equilibrium fluctuations in an originally unperturbed sample. A first question that arises is how the growth of the fluctuations is related to the development of a macroscopic concentration profile, starting from an originally uniform concentration. As we will see shortly, a meaningful parameter reflecting the growth of the macroscopic state is the concentration difference $\Delta c(t)$ generated by the Soret flow across the sample. This quantity can be calculated theoretically from Eqn. 2:

$$
\begin{aligned}
& \Delta c(t)=c(0, t)-c(1, t)= \\
& =a \nabla c_{s}\left\{1-\frac{4}{\pi^{2}} \sum_{j=1}^{\infty} \frac{1}{j^{2}}\left[1-(-1)^{j}\right] \times \exp \left(-\frac{j^{2} \pi^{2} D}{a^{2}} t\right)\right\}
\end{aligned}
$$

In Fig. 2 we have superimposed the time evolution of the concentration difference $\Delta c(t)$ to the experimental results for the variance of the fluctuations. The dashed line is obtained from Eqn. 8 using the reference value for $D$ [45], the only free parameter being a multiplicative constant, while the solid line is a fit obtained by also using the diffusion coefficient as a free parameter. One can appreciate that the growth of $\Delta c(t)$ is in good agreement with the growth of the variance of the shadowgraph images. Therefore, the variance of the non-equilibrium fluctuations at the large length scales where the fluctuations are strongly affected by gravity is intimately related to the dynamics of growth of the concentration difference, which in turn allows the determination of the diffusion and Soret coefficient. Although this method for the determination of the diffusion coefficient is very preliminar, this approach, when properly developed, could represent a suitable alternative to Mach-Zehnder Interferometry, Electronic Speckle Pattern Interferometry or Beam Deflection to recover the transport coefficient of a binary liquid mixture during a thermodiffusion experiment.

A more refined assessment of the kinetics of growth of non-equilibrium fluctuations involves the determination of their structure factor, which is directly related to the structure factor of the shadowgraph images $S_{S}(q, t)$, where $q$ is the transferred wave vector:

$$
S_{S}(q, t)=T(q)\left[S_{T}(q, t)+S_{c}(q, t)\right]+S_{D}(q, t)
$$

where $S_{T}(q, t)$ and $S_{c}(q, t)$ are the power spectra of temperature and concentration fluctuations respectively, $S_{D}(q, t)$ is the power spectrum of the dynamic camera noise and $T(q)$ is the transfer function of the shadowgraph method $[7,23,15,47]$ 


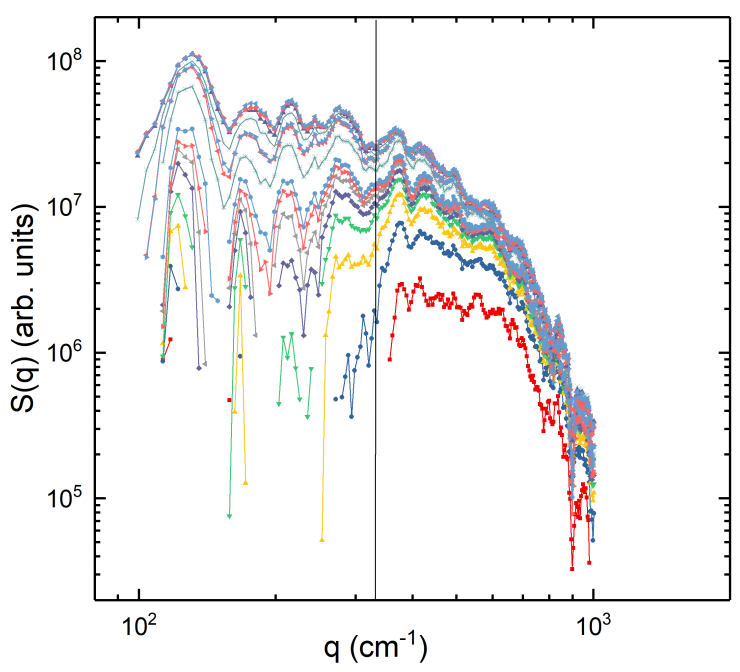

Fig. 3. Time evolution of the static structure factor $T(q) S_{c}(q, t)$ of non-equilibrium concentration fluctuations taken during the transient phase. The first curve (lower one) is taken at $t=180 \mathrm{~s}$ after the imposition of the temperature gradient, while the last one at $t=3600 \mathrm{~s}$. The solid vertical line shows the theoretical roll-off wave vector $q_{R O}$ at steady state.

In order to compare our results with the data presented in Ref. [15] we study the structure factor of the images at various time. To get rid of the contribution of the temperature fluctuations and of the dynamic camera noise we evaluate the power spectrum $S_{0}(q)$ of the shadowgraph images grabbed about 100s after the imposition of the gradient. Due to the large separation of the timescales for the growth of fluctuations, after this time the temperature fluctuations are fully developed, while the concentration fluctuations have barely started to grow and $S_{0}(q)=T(q) S_{T}(q, t)+S_{D}(q, t)$. The subtraction of $S_{0}(q)$ from $S_{S}(q, t)$ allows to isolate the contribution of nonequilibrium fluctuations, (Fig. 3):

$$
S_{S}(q, t)-S_{0}(q)=T(q) S_{c}(q, t)
$$

From Fig. 3 we observe that the structure factor grows rapidly in the first hour, and slows down during the following part of the process. To characterize the transient regime we analyzed, for various runs, the first 900 images collected every 4 seconds after applying the temperature difference across the cell.

The typical oscillations due to the shadowgraph transfer function are visible, superimposed to the static structure factors [40]. As the signal due to non-equilibrium concentration fluctuations is very small during the early stages of growth, data are particularly noisy at small wave vectors, and it is difficult to make any statement on the presence of a peak predicted in Ref. [15].

From Eqn. 1, we can estimate the steady state roll off for our sample to be $q_{R O}=334 \mathrm{~cm}^{-1}$ (being $\beta=0.373$, $\nu=4.91 \times 10^{-2} \mathrm{~cm}^{2} / \mathrm{s}, \nabla c_{s}=0.71 \mathrm{~cm}^{-1}$, and $D=$ $\left.4.3 \times 10^{-7} \mathrm{~cm}^{2} \mathrm{~s}^{-1}\right)$. Its position is made evident in Fig. 3 with the straight vertical line. $q_{R O}$ seems slightly underestimated with respect to the actual roll off position for all the curves. Actually Eqn. 1 is obtained in the hypothesis of dilute samples and at the stationary state, when a constant gradient is present in the cell, and a vertical displacement of a volume of sample creates a density mismatch.

The time evolution of the structure factors shown in Fig. 3 is directly related with the evolution of the variance of shadowgraph images shown in Fig. 2. More in detail, the integral of the structure factor at a given time over the accessible $q$-range is directly proportional to the variance of the shadowgraph images. A more refined analysis involves the experimental determination of the growth rate as a function of wave vector of the structure factor of the fluctuations $S_{c}(q, t)$ :

$$
\begin{array}{r}
R_{f}(q, t)=\frac{S_{S}(q, t+\Delta t)-S_{S}(q, t)}{\left[S_{S}(q, t)-S_{0}(q)\right] \Delta t}= \\
=\frac{S_{c}(q, t+\Delta t)-S_{c}(q, t)}{S_{c}(q, t) \Delta t}
\end{array}
$$

As it can be appreciated from Eqn. 11, this method for analysing the kinetics of growth of the fluctuations allows to get rid completely of the shadowgraph transfer function without the need of performing calibration measurements. By combining equations 5 and 11 one finds the remarkable result that the growth rate $R_{f}$ of the structure factor of the fluctuations as a function of wave vector is exactly equal to the growth rate $R_{\Delta c}$ of the concentration difference $\Delta c$. Using Eq. 2 and 11 we can evaluate theoretically the growth rate of the concentration difference $\Delta c$ as

$$
\begin{aligned}
& R_{\Delta c}(t)=\frac{\Delta c(t+\delta t)-\Delta c(t)}{\Delta c(t) \delta t}= \\
& =\frac{4 D \sum_{j=1}^{\infty}\left[1-(-1)^{j}\right] \exp \left(-j^{2} \pi^{2} \frac{D}{a^{2}} t\right)}{a^{2}\left[1-\frac{4}{\pi^{2}} \sum_{j=1}^{\infty} \frac{1}{j^{2}}\left[1-(-1)^{j}\right] \exp \left(-j^{2} \pi^{2} \frac{D}{a^{2}} t\right)\right]}
\end{aligned}
$$

In Fig. 4 we show the comparison between the time evolution of the growth rate of fluctuations defined in Eqn. 11 for different wave vector ranges.

At variance to what expected in a diffusive process, where the growth of the amplitude of the concentration modes is strongly dependent on the wave vector $q$, being $c(q, t) \propto\left[1-\exp \left(-D q^{2} t\right)\right]$, we notice that the data for the growth rate of non-equilibrium fluctuations approximately collapse onto the same curve, without evidence of any dependence on the particular $q$ value.

Figure 4 shows the superposition of the growth rate of fluctuations and the rate theoretically calculated from the concentration profile as indicated in Eqn. 12 and the experimental rate of growth of non-equilibrium concentration fluctuations. The dashed line is obtained from Eq.12 


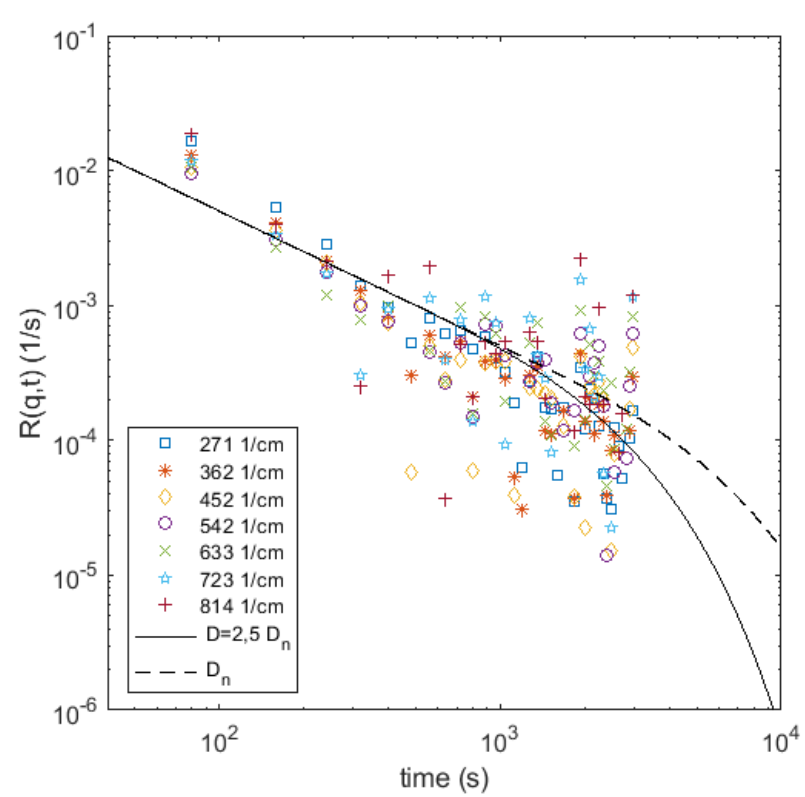

Fig. 4. Time evolution of the growth rate of non-equilibrium concentration fluctuations for different ranges of wave vectors. The two lines are calculated using Eqn. 12 without adjustable parameters: the dashed line with the nominal diffusion coefficient $D_{n}$ while the solid line with $D=2.5 D_{n}$. One can appreciate that the growth rate during the onset of the fluctuations is independent on the diffusion coefficient, that becomes significant only at large times, as shown by the theoretical curves.

without any adjustable parameters and using the nominal diffusion coefficient $\left(D=4.3 \times 10^{-7} \mathrm{~cm}^{2} \mathrm{~s}^{-1}\right)$, while the solid line is obtained using the value of the diffusion coefficient recovered from the fitting of the variance shown in Fig. $2\left(D=10.8 \times 10^{-7} \mathrm{~cm}^{2} \mathrm{~s}^{-1}\right)$. One can appreciate that at small $t$ the rate of growth does not show the presence of any typical time scale and follows a powerlaw behavior $R_{c}(q, t) \propto 1 / t$. This behavior is independent from the value of the diffusion coefficient. At larger $t$, the finite thickness of the sample determines a cut-off, which strongly depends from the value of the diffusion coefficient.

The overall good agreement between the growth rates shown in Fig. 4 confirms the theoretical result outlined above that the growth of fluctuations at the mesoscopic scale is directly related to the development of the concentration profile at the macroscopic scale. A more complete and refined understanding of the kinetics of growth of non-equilibrium concentration fluctuations would require performing experiments under microgravity conditions, where the amplitude of non-equilibrium fluctuations at small wave vectors is not limited by the presence of gravity. This represents one of the main goals of the GiantFluctuations (NEUF-DIX) project of the European Space Agency, which will be hosted on the International Space Station during the next years [21].

\section{Acknowledgements}

We thank W. Köhler and J. M. Ortiz de Zárate for useful discussion. Work partially supported by the European Space Agency.

\section{Author contributions}

M. C., F. C., and A. V. conceived the study. M. S. performed measurements. M. C., M. S. and A. V. analyzed results. M. C. and A. V. wrote the paper. All the authors contributed to the discussion of the results.

\section{References}

1. J.M. Ortiz de Zárate, J.V. Sengers, Hydrodynamic fluctuations in fluids and fluid mixtures (Elsevier, Amsterdam, 2006)

2. F. Croccolo, J.M. Ortiz de Zárate, J.V. Sengers, Eur. Phys. J. E 39, 125 (2016)

3. P.N. Segrè, R.W. Gammon, J.V. Sengers, Phys. Rev. E 47, 1026 (1993)

4. A. Vailati, M. Giglio, Phys. Rev. Lett. 77, 1484 (1996)

5. A. Vailati, M. Giglio, Progr. Colloid Polym. Sci. 104, 76 (1997)

6. D. Brogioli, A. Vailati, M. Giglio, Phys. Rev. E 61, R1 (2000)

7. F. Croccolo, D. Brogioli, A. Vailati, M. Giglio, D.S. Cannell, Phys. Rev. E 76, 041112 (2007)

8. F. Giavazzi, G. Savorana, A. Vailati, R. Cerbino, Soft Matter 12, 6588 (2016)

9. S. Bonella, M. Ferrario, G. Ciccotti, Langmuir 33, 11281 (2017)

10. A. Mialdun, C. Minetti, Y.Gaponenko, V. Shevtsova, F. Dubois, Microgravity Sci. Technol. 25, 83 (2013)

11. V. Shevtsova, C. Santos, V. Sechenyh, J.C. Legros, A. Mialdun, Microgravity Sci. Technol. 25, 275 (2018)

12. D.A. de Mezquia, M. Larrañaga, M.M. Bou-Ali, J.A. Madariaga, C. Santamaría, J.K. Platten, Int. J. Therm. Sci. 92, 14 (2015)

13. T. Triller, H. Bataller, M. Bou-Ali, M. Braibanti, F. Croccolo, J. Ezquerro, Q. Galand, J. Gavald, E. Lapeira, A. Lavern-Simavilla et al., Microgravity Sci. and Technol. 30, 295 (2018)

14. A. Vailati, M. Giglio, Phys. Rev. E 58, 4361 (1998)

15. R. Cerbino, Y. Sun, A. Donev, A. Vailati, Sci. Rep. 5, 14486 (2015)

16. J.S. Huang, W.I. Goldburg, A.W. Bjierkaas, Phys. Rev. Lett. 32, 921 (1974)

17. K. Binder, D. Stauffer, Phys. Rev. Lett. 33, 1006 (1974)

18. H.A. Furukawa, Adv. Phys. 34, 703 (1985)

19. M. Carpineti, M. Giglio, Phys. Rev. Lett. 68, 3327 (1992)

20. F. Croccolo, H. Bataller, Eur. Phys. J. E 39, 132 (2016)

21. P. Baaske, H. Bataller, M. Braibanti, M. Carpineti, R. Cerbino, F. Croccolo, A. Donev, W. Köhler, J.M. Ortiz de Zárate, A. Vailati, Eur. Phys. J. E 39, 119 (2016)

22. S.P. Trainoff, D.S. Cannell, Phys. Fluids 14, 1340 (2002)

23. A. Vailati, R. Cerbino, S. Mazzoni, C.J. Takacs, D.S. Cannell, M. Giglio, Nature Comm 2, 290 (2011)

24. D. Brogioli, A. Vailati, Phys. Rev. E 63, 012105 (2001) 
25. T.R. Kirkpatrick, J.M. Ortiz de Zárate, J.V. Sengers, Phys. Rev. Lett. 110, 235902 (2013)

26. T.R. Kirkpatrick, J.M. Ortiz de Zárate, J.V. Sengers, Phys. Rev. Lett. 115, 035901 (2015)

27. J.M. Ortiz de Zárate, T.R. Kirkpatrick, J.V. Sengers, Eur. Phys. J. E 38, 99 (2015)

28. T.R. Kirkpatrick, J.M. Ortiz de Zárate, J.V. Sengers, Phys. Rev. E 93, 012148 (2016)

29. T.R. Kirkpatrick, J.M. Ortiz de Zárate, J.V. Sengers, Phys. Rev. E 93, 032117 (2016)

30. D. Ronis, I. Procaccia, Phys. Rev. A 26, 1812 (1982)

31. B.M. Law, J.C. Nieuwoudt, Phys. Rev. A 40, 3880 (1989)

32. J.C. Nieuwoudt, B.M. Law, Phys. Rev. A 42, 2003 (1990)

33. D. Brogioli, F. Croccolo, A. Vailati, Phys. Rev. E 94, $022142(2016)$

34. P.N. Segré, J.V. Sengers, Physica A 198, 46 (1993)

35. W.B. Li, P.N. Segré, R.W. Gammon, J.V. Sengers, Physica A 204, 399 (1994)

36. W.B. Li, P.N. Segré, R.W. Gammon, J.V. Sengers, J. Phys.: Condens. Matter A 6, 119 (1994)

37. A. Vailati, M. Giglio, Nature 390, 262 (1997)

38. W.B. Li, K.J. Zhang, J.V. Sengers, R.W. Gammon, J.M. Ortiz de Zárate, Phys. Rev. Lett. 81, 5580 (1998)

39. F. Giavazzi, A. Fornasieri, A. Vailati, R. Cerbino, Eur. Phys. J. E 39, 103 (2016)

40. F. Croccolo, C. Giraudet, H. Bataller, R. Cerbino, A. Vailati, Microgravity Sci. Technol. 28, 467 (2016)

41. H. Bataller, C. Giraudet, F. Croccolo, J.M. Ortiz de Zárate, J. M., Microgravity Sci. Technol., accepted for publication (2016)

42. C. Giraudet, H. Bataller, Y. Sun, A. Donev, J.M. Ortiz de Zárate, F. Croccolo, Europhys. Lett. 111, 60013 (2015)

43. J. Crank, The Mathematics of Diffusion (Oxford University, Oxford, 1975)

44. C. Tanford, Physical Chemistry of Macromolecules (Wiley, New York, 1961)

45. K. Zhang, M.E. Briggs, R.W. Gammon, J.V. Sengers, J.F. Douglas, J. Chem. Phys. 111, 2270 (1999)

46. J. Rauch, W. Köhler, J. Phys. Chem. 119, 11977 (2003)

47. F. Croccolo, D. Brogioli, App. Opt. 50, 3419 (2011) 